\title{
X-FILEs Jam: Ideation Process and Outcomes
}

\author{
Lawrence C. Ragan and Lorraine J. Ramirez Villarin
}

\section{Motivations}

In exploring the future of STEM education in 2026, the research team recognized the importance of gaining insights from a variety of stakeholders including administrators, faculty, instructional design and technology professionals, and visionaries who scan and identify trends and forces affecting higher education. Perhaps the most critical perspectives, however, were the insights, thoughts, opinions, and hopes of the user population, the students. The 2018 X-FILEs Workshop included a student-led discussion panel providing their perspective of STEM education in 2026. Four students, representing high school through graduate students, served as panelists and shared their hopes and expectations of the higher education experience. The X-FILEs research team recognized the value of this panel to the X-FILEs project and determined to structure an event that would stimulate and harness ideas directly from undergraduates and graduates. The target population was expanded from students representing STEM fields to students representing a range of backgrounds and academic disciplines.

The purpose of the student-based event was to gain complementary input that would enhance the data gathered at the 2018 X-FILEs Workshop. The form and structure of the 2018 Workshop would not be appropriate as it did not match the interest and logistics of a 1-day format. The project team settled on the "jam" model of ideation to organize and operate the event. Jams, sometimes referred to as "ideathons" or "designathons," focus on idea development rather than the produc-

\author{
L. C. Ragan (四) \\ Ragan Education, State College, PA, USA \\ e-mail: 1cr1@psu.edu \\ L. J. Ramirez Villarin \\ University of North Georgia, Dahlonega, GA, USA \\ e-mail: Lorraine.RamirezVillarin@ung.edu
}




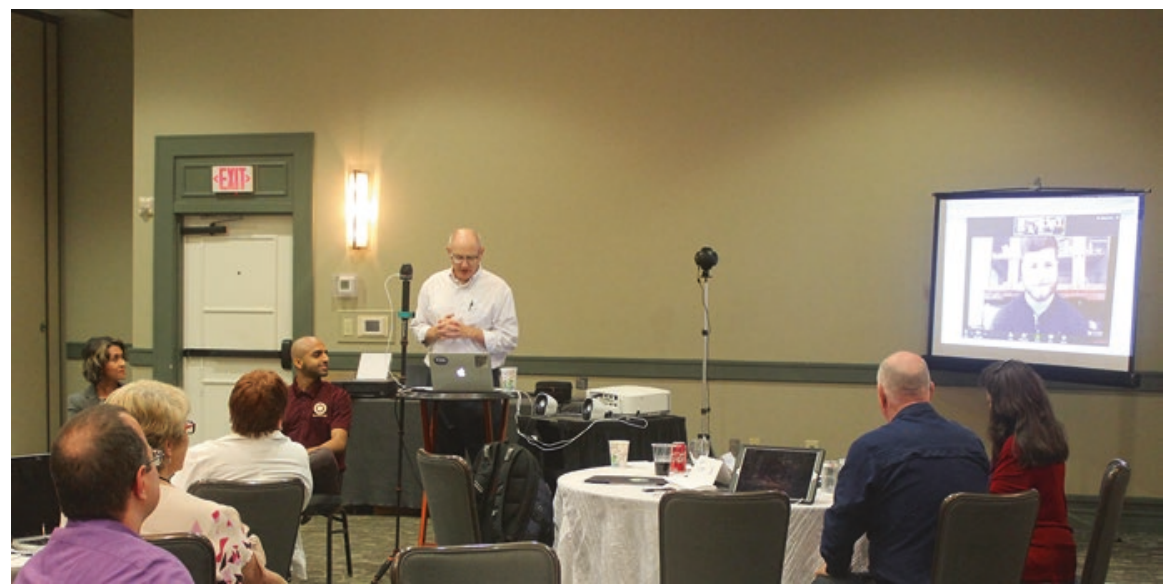

Fig. 1 X-FILEs Student Panel Session

tion of a product. Jams are team-based, loosely structured exercises conducted in a face-to-face environment designed to bring out participants' creativity for developing innovative solutions to complex problems (Morrison 2009).

Jams engage students and promote creativity by giving them a challenge statement and structured guidance for solving it. The most creative jams require a multidisciplinary team in order to achieve the desired outcomes. Challenges require participants to apply knowledge to solve real-world problems. The X-FILEs Jam focused on a modified challenge statement derived from the 2018 X-FILEs Workshop (Fig. 1).

"Using what you've learned about innovative learning environments, create a solution that improves or enhances the student experience for a challenging dimension of college-level STEM education."

\section{Goals}

Applying the challenge statement to the X-FILEs Jam program, the goal was to generate a collection of student idea-solutions where one or more of four technology categories may be applied to an aspect of teaching and learning to address a specific problem in STEM education. During the process, the research team gathered the participants' ideas and brainstorming results through a series of written tasks to track common patterns and keep a record of students' thoughts. Findings reflected what students envisioned of their STEM education experience in the near future. 


\section{Program Summary}

\subsection{Technology and Teaching and Learning Framework}

In order to maintain connective themes with the original 2018 X-FILEs Workshop, the same four technology categories and the aspects of teaching and learning with their definitions were incorporated. Technology categories included personalized/ adaptive learning, multimodal learning, cross reality, and artificial intelligence/ machine learning. The aspects of teaching and learning encompassed content presentation, interactions and communications, learner activities, assessment, and cocurricular activities. The student teams were encouraged to address at what point in the course experience these technologies (individually or a creative mix) may be applied. The project's principal investigators, Drs. Ryoo and Winkelmann presented the definition and descriptions of these categories as they applied to the Jam. A video recording is available on the Jam website (Ryoo n.d.).

\subsection{Design}

Students, working in teams of three or four individuals, were guided through the day's ideation process in order to facilitate the successful completion of their ideasolution addressing the X-FILEs Jam challenge statement. Team idea-solutions were incubated and developed over a period of 6 to $8 \mathrm{~h}$ and presented at the conclusion of the Workshop. Award categories and prizes were described at the beginning of the program with the final awards being presented during the wrap-up activity.

\subsection{Jam's Summary of Events}

The X-FILEs Jam began with a tour and hands-on experiences guided by Martin Gallagher, director of the Evans Library Digital Scholarship Lab on the Florida Tech campus. He introduced the students to the four categories of emerging technologies discussed in the 2018 Workshop. This presentation was followed by participants exploring, interacting with, and reflecting on how today's technologies can become vital resources on future STEM higher education instruction.

Lawrence C. Ragan, the day's facilitator, introduced the X-FILEs research team and sponsors, followed by a review of the events, timing, and desired outcomes. The principal investigators, Drs. Ryoo and Winkelmann gave presentations to familiarize students with the transformative technologies in STEM education featured in the 2018 X-FILEs Workshop. Definitions and the potential of each technology, as well as examples, were provided.

The "STEM Idea Harvest" involved students in a gallery walk activity with stations representing each of the five aspects of teaching and learning. As the students 


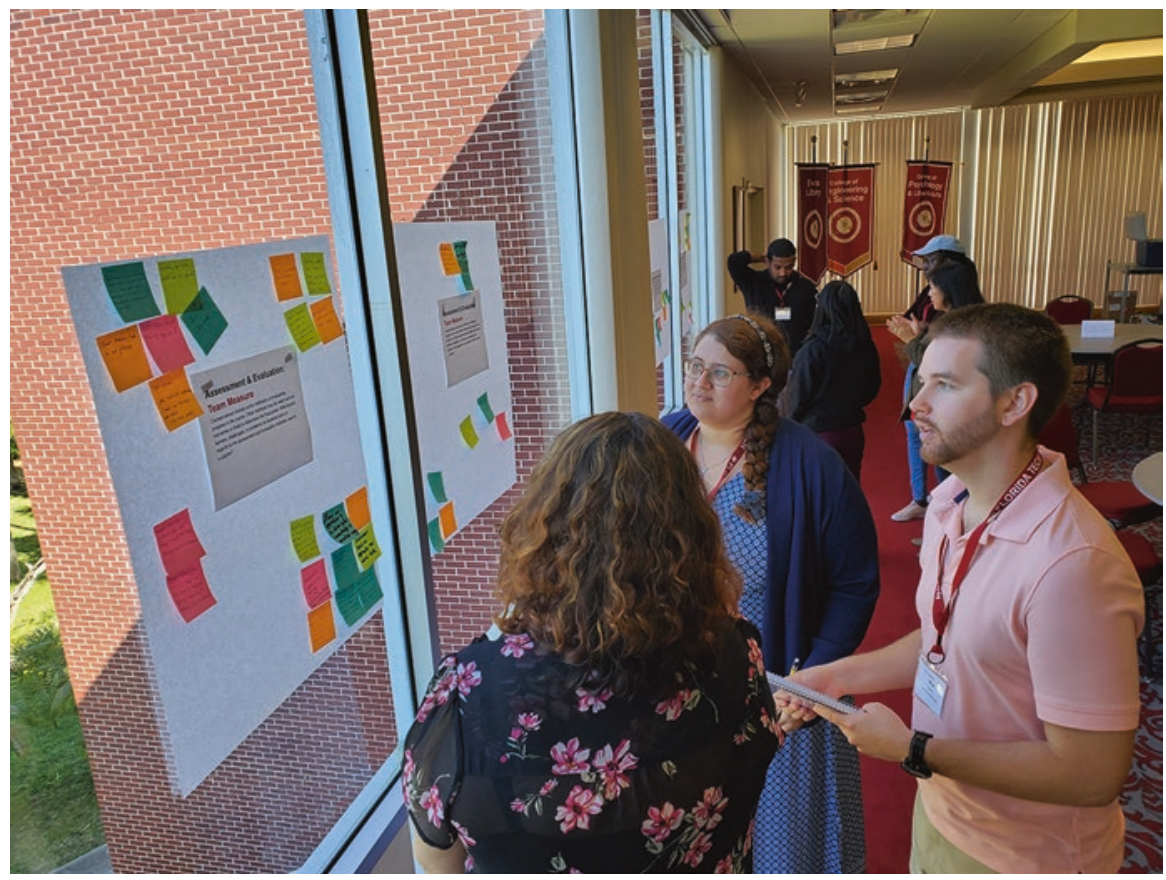

Fig. 2 Jam participants engaging in the gallery walk activity at the assessment station. February 8,2020

arrived at each station, they reflected on the challenges, barriers, and opportunities presented by that aspect. They wrote their many ideas on sticky notes at each station. Students moved to all five stations in 3- to 5-min increments (Fig. 2).

In order to form the day's teams, participants were asked to select one of the aspects based on their personal interests. The desire was to form teams with a mix of discipline interests and levels of undergraduate or graduate education. Participants organized into five teams:

- Team Stuff representing content presentation

- Team WhatsApp representing interactions and communications

- Team To-Do representing learner activities

- Team Measure representing assessment remove evaluation

- Team Extra Extra representing co-curricular activities

Team members introduced themselves and developed a "How Might We ..." starting statement for their team-based on the generic example:

"How might we improve learning for students to enjoy, embrace, and apply STEM concepts using a variety of existing and future technologies?"

The day's agenda consisted of a series of activities designed to promote team formation, ideation, solution creation, and idea refinement. The beginning activity was the "Innovation Disruption Analysis." Teams randomly selected three cards from the Business Innovations Cards stack (Board of Innovation 2020). Each card 
provided an example of a case study from a company that generated a new product after recognizing a gap in the industry. The cards represented the company's responses to technological, market, customer, and regulation trends. Teams reflected on why it was an inspiring case and how it became an innovative disruption. They later presented a short description on how these disruptions may influence their idea-solution. The facilitators declared Team To-Do, focusing on the aspect of learner activities, winners after suggesting how the Duolingo app could be incorporated into each of the aspects of teaching and learning to improve the students' learning experience.

Participants continued with an "opposite thinking" exercise. Teams developed a list of assumptions for their aspect of teaching and learning. Based on these assumptions, the teams formulated the opposite response or representation of the assumption. For example, if one assumption of STEM education is that all students receive homework assignments using the same context, an opposite response may be that the context is personalized for each student based on their personal interest or area of study. Each team was provided writing materials and encouraged to record their ideas and the process as they developed their idea-solution plans.

To conceptualize the idea-solution, teams engaged in the "Amazing Idea-Solution Process." Inspired by the show The Amazing Race, the task involved teams independently completing certain steps to narrow their ideas before moving forward (Fig. 3). Five cards were created with instructions on how to complete each steps. The facili-

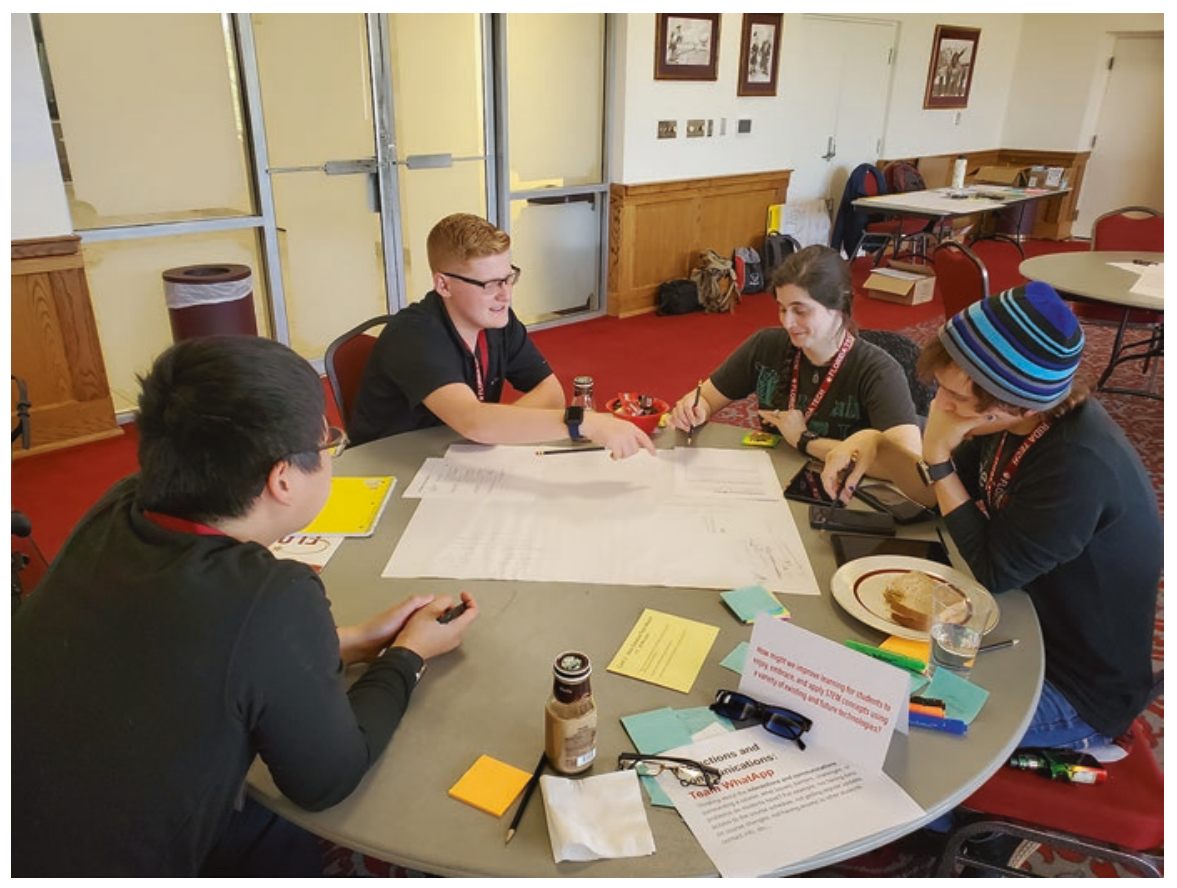

Fig. 3 Participants from Team WhatsApp working on Card 2 of the "Amazing Idea-Solution Process.” February 8, 2020 
tators provided the next level card to teams after step completion. These cards for the idea-solution development were:

Card 1. Solution identification

Card 2. Idea-solution/tech match

Card 3. Idea-solution user

Card 4. Amazing idea development

Card 5. Present with confidence!

At the completion of the "Amazing Idea-Solution Process," teams presented their idea-solutions to the larger community in a 10-min presentation and a 2-min question and answer session. While the participants voted for the Student's Choice Award, five judges evaluated the presentations using a criteria checklist to determine the winners in the remaining categories. Drs. Ryoo and Winkelmann presented the awards which would earn a gift card to each student in a team:

1. Best overall

2. Best technology

3. Most creative and innovative

4. Most impactful

5. Student choice

\section{Outcomes}

\subsection{Trends Generated from the STEM Idea Harvest}

The future of innovative learning environments within each aspect of teaching and learning presented challenges, barriers, or problems for students. Many of these were self-evident, such as the high cost of study materials or the lack of access to digital class notes. Others were more subtle and may only affect certain individuals or populations, such as students with physical or emotional disabilities and access to class materials while being deployed in the military. The following list represents a sampling of the recurring themes captured from the participants' notes.

Content Presentation Context Presented to Students: "When considering how the course content is managed and taught in a course, what issues, barriers, challenges, or problems do students have? For example, it may be the cost of materials, the timing of when you can get the materials or even the weight of the books! Include in this question the "teaching" of this course material. For example, too many lectures, not enough review time, lack of access to the professor, etc."

Common Themes of Participant Input:

- Time-Concerns were expressed around the impact of too little or too much time on a course. In general, when a set timeframe is established for all students, some 
will feel rushed through the materials, while others become impatient to move ahead. This perennial dilemma causes gaps in understanding or inhibits the ability to fully apply content knowledge within a real-life perspective.

- Cost-Participants identified the cost of a course and materials as a barrier to their educational experience. Some online programs may require additional subscriptions with fees not usually covered by the university.

- Resources-Participants also expressed a need for alternative sources of content, as textbooks quickly become outdated and obsolete. Additionally, course content may not always be available in multiple formats to accommodate students with disabilities or special needs.

- Relevancy-Students' most recurrent problem was the lack of relevance between course content and real-world tasks and applications.

- Additional suggestions included adapting the course content to the time allotted, consolidating material, personalizing or customizing content to students' needs, and making a more engaging or hands-on learning experience.

Interactions and Communications Context Presented to Students: “Thinking about the interactions and communications surrounding a course, what issues, barriers, challenges, or problems do students have? For example, not having easy access to the course schedule, not getting regular updates on course changes, not having access to other students' contact info, etc."

Common Themes of Participant Input:

- Time restrictions - There is limited access for individual meetings with instructors during office hours. One professor may not have time for all of his/her students.

- Communication anxiety or barrier-Students expressed concerns that communication and understanding may be affected when there is "contact apprehension" between teacher and student due to culture, language, power difference, or social anxiety.

- Lack of consistency-Other communication and interaction problems identified included the absence of consistency of style and format among educators. Different faculty use different methods, tools, and styles to interact with their students. This inconsistency of feedback from instructors makes it difficult for students to navigate course activities. Participants believe that learner and educator expectations are different and lack to portray real-world outcomes.

- Suggestions for improvement included the mandated use of Learning Management Systems, standardized versions of syllabi formats, and improved access to instructors.

- Participants also suggested providing increased customization of the student portal (e.g. individualized course interface).

Learner Activities Context Presented to Students: "The student activities include course assignments or requirements that encourage students to apply, practice and further develop facility with the course objectives. What issues, barriers, challenges, 
or problems do students face in this aspect of the course? For example, lack of time to complete assignments, no support available when needed, etc...."

Common Themes of Participant Input:

- Response Timeliness-Students expressed frustration with the lack of assistance and feedback when needed. Students related a desire for 24-hour help, especially for distance learners.

- Relevance-The second most common complaint was the absence of learner activities with real-life applications or relevance.

- Guidance/instructions-Suggestions involved designing clearer instructions and guidance on assignments.

- Engaging Activities-Students expressed a desire for more engaging activities that incorporate technology (e.g. VR, apps) and work related to the field of study.

- Suggestions included making learner activities more relatable to students' hobbies and backgrounds where possible. Another suggestion was for activities to be compliant with learner's pace and more collaborative work to reduce homework time.

Assessment Context Presented to Students: "Courses always include some method(s) of assessing progress in the course. Other methods may be used such as midterms or finals to determine the final grade. What issues, barriers, challenges, or problems do students have in regards to the assessment and evaluation methods used in a course?"

\section{Common Themes of Participant Input:}

- Testing Environment-Current evaluation and assessment strategies do not frequently take into account the uniqueness of each individual. Treating all students the same eliminates the ability to allow students with differences to show what they are capable of accomplishing.

- Fairness-Participants proposed adjusting grading on an as-needed basis by the instructor, for example, adding a curve scale when necessary or using equal weighting.

- Suggested alternative assessments comprised the use of tests based on real-world scenarios, making more personalized assessments based on student experiences and incorporating VR and other technology assessments to decrease costs and promote a more ethical curriculum (e.g., eliminating real-life animal dissections). Other suggestions included simple adjustments as modifying the testing environment, allowing additional time, and assessing skills rather than rote memory.

Co-curricular Activities Context Presented to Students: "Many times courses will include materials or experiences beyond the scope of the actual course. These may be related to the course content while other times they are "nice to haves" but not critical. For example, attending a lecture or presentation given by a visiting scholar or joining the BioResearch Club. What issues, barriers, challenges, or problems do students have with co-curricular activities?"

Common Themes of Participant Input: 
- Limited Access/Lack of Resources-For some students, the distance to club meetings and activities (e.g., field trips) is not within reasonable limits. Virtual reality may allow remote participation, but not everybody has access to the equipment.

- Cost-Engaging in extracurricular activities can place an increased cost burden on the student that inhibits their participation. In some cases these activities are mandated (e.g., conferences, field trips) or highly recommended, and the student may not have the funds to participate.

- Scarce Industry Opportunities-identifying suitable opportunities to engage with intern programs is challenging with limited access to data. Students need flexibility and agility in finding a program that fits their academic and personal circumstances.

- Time-As students are increasingly occupied with work, family, and academic activities, finding the time to participate in extracurricular relationships presents a barrier.

\subsection{Amazing Idea-Solutions}

Five teams of three or four individuals were organized as an output of the aspects of teaching and learning Idea Harvest. Each team chose a single aspect to use as the context for their idea-solution. The participants' thoughts garnered on post-it notes through the gallery walk became the basis for the day's work. The remaining sequence of the day's events guided the teams through the ideation process in order to present a final idea-solution for the Jam competition.

Content Presentation Team Stuff designed a "Fitbit"-type device that could track a student's learning pace and could suggest "learning time" that an individual should devote to a course or task.

Interactions and Communications Team WhatsApp conceived an app that would connect students 24-hours a day with either live or AI tutors worldwide to provide assistance clarifying content or requiring help related to course materials.

Learner Activities Team To-Do addressed a modification to Learning Management Systems via a survey to customize student's learning activities to their hobbies, interests, or careers making them more meaningful and engaging. This idea was premised on the belief that students would be more interested and motivated with their learning when it directly interacted with their personal interests.

Assessment Team Measure's idea consisted of creating a "controlled virtual reality environment" for administering formative assessments to students requiring special accommodations (e.g. ambient music and relaxing atmosphere, tasks assessed virtually, track eye movement with Oculus to denote attention, etc.) 
Co-curricular Activities Team Extra Extra formulated an app called "Mentor Tinder." This app would consist of a database capable of matching a student with appropriate mentors that shared their interests and expectations. These mentor relationships could be local, regional, or even international.

\section{Recommendations}

The X-FILEs Jam was considered a success in gathering input from the end users, the students. There are features of the event that, slightly modified, may have produced different outcomes. The research team makes the following recommendations seeking to improve the experience for students as well as more closely align with the original X-FILEs project goals.

1. The challenge statement used as the basis of the Jam focused the student team's efforts on designing an idea-solution to a specific problem in STEM education. A modification to the Jam challenge statement to more closely align with the statement used for the X-FILEs Workshop would be beneficial in producing complementary outcomes from the Jam experience.

2. Leading individuals through the ideation process requires time for reflection and deeper thinking. Recommended strategies to enable additional "incubation" time for teams to consider new or novel idea-solutions would include forming teams as an outcome of pre-Jam webinars and enabling discussion/forum boards to encourage interaction prior to the Jam event.

3. Predictably, without adequate encouragement and time, teams gravitated toward "top-of-mind" and obvious idea-solutions. Including a process to share and review early ideas with program organizers or other guests would guide teams toward more new or novel solutions.

4. Conduct pre-Jam events including an online design competition in order to stimulate creative thinking and jumpstart the idea-solution development at the event.

5. Establish at least two teams working on the same dimension of an aspect of teaching and learning. At the appropriate time, combine these teams into one in order to generate a new and novel idea-solution.

6. Require teams to conduct research or perform literature reviews to determine if the idea-solution may already exist.

7. Provide teams with access to the technologies they have employed in order to test the feasibility of in their idea-solution.

8. Place increased emphasis on the unique and innovative nature of the desired outcomes throughout the Jam events.

9. Increase the number of participants through a variety of strategies including introducing the team competition during the pre-Jam webinars (Fig. 4). 


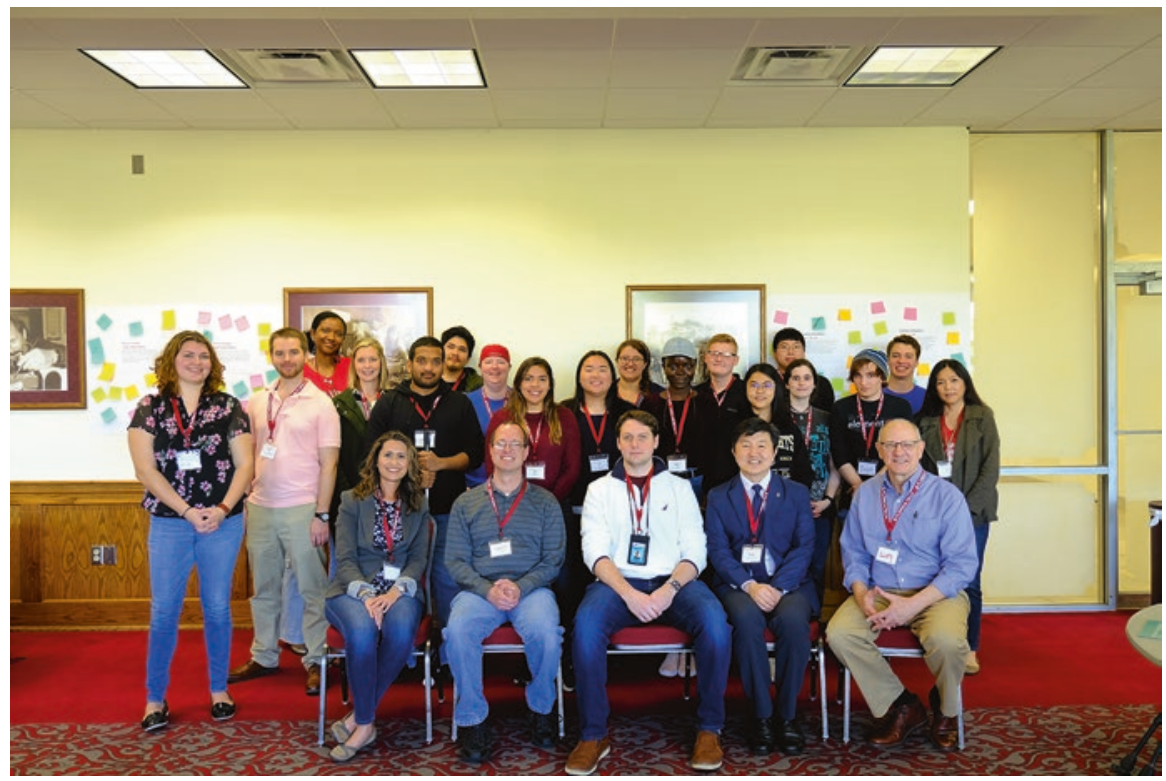

Fig. 4 X-FILEs Jam Organizers and Participants

\section{Conclusion}

Gaining the insights and input of the end user of any system is a critical step in product design. Soliciting the student voice in describing and envisioning the future of STEM education through a Jam event proved to be an effective and educational experience for all. The success of the program was largely due to the willingness of the participants to address the challenge by generating creative and practical idea-solutions. Although the number of student participants was less than anticipated from preregistration data, they dedicated the day to working as a team to consider how to improve the state of STEM education through the use of the four technology categories. The quality of their presentations of idea-solutions validated the process and the efforts of each team.

Capturing the student output through the transcription of notes and drawings provided a glance into the ideation progress and outcomes from each team. The positive interactions among participants and their willingness to consult with the $\mathrm{X}$-FILEs research team served as a valuable guiding force throughout the event. Final evaluations denoted a high sense of satisfaction by students, suggesting the participants enjoyed and found value in the day's activities. Evaluations encompassed everything from the accommodations to the facilitators and the overall view of the event. 
As in every event offering, reflection on the areas for improvement presents an opportunity to enhance and enrich the event design. The recommendations suggest minor adjustments to a Jam event in order to more closely align the designed activities to the larger program goals.

\section{References}

Board of Innovation (2020) Brainstorm cards. https://www.boardofinnovation.com/tools/ brainstorm-cards

Morrison K (2009) The implications of 'jam' and other ideation technologies for organisational decision making. Cult Sci Journal 2(1):1. https://doi.org/10.5334/csci.21

Ryoo J (n.d.) eXploring the Future of Innovative Learning Environments (X-FILEs) JamStudent-generated solutions to challenges in STEM higher education. https://sites.psu.edu/ filejam/. Accessed 6 Apr 2020

Open Access This chapter is licensed under the terms of the Creative Commons Attribution 4.0 International License (http://creativecommons.org/licenses/by/4.0/), which permits use, sharing, adaptation, distribution and reproduction in any medium or format, as long as you give appropriate credit to the original author(s) and the source, provide a link to the Creative Commons license and indicate if changes were made.

The images or other third party material in this chapter are included in the chapter's Creative Commons license, unless indicated otherwise in a credit line to the material. If material is not included in the chapter's Creative Commons license and your intended use is not permitted by statutory regulation or exceeds the permitted use, you will need to obtain permission directly from the copyright holder. 